\title{
Effect of portal-systemic anastomosis on renal haemodynamics in cirrhosis
}

\author{
H. RING-LARSEN, B. HESSE, AND B. STIGSBY \\ From the Division of Hepatology, Medical department A, Cardiovascular Laboratory, Medical \\ Department B, Rigshospitalet, University Hospital, Copenhagen, and Department of Surgical \\ Gastroenterology, Copenhagen County Hospital in Gentofte, Copenhagen, Denmark
}

SUMMARY In 12 patients with portal hypertension and repeated bleedings from oesophageal varices the central haemodynamics, portal pressure, and mean renal blood flow (RBF) were investigated immediately before and two to seven months after portal-systemic shunt. Cardiac output increased significantly, whereas arterial pressure was unchanged after operation. RBF, which was initially less than in controls, did not change. As portal pressure decreased significantly, a direct portalrenal, neural, or humoral reflex mechanism does not explain the subnormal RBF in cirrhosis. As plasma volume was large and unchanged after operation a 'diminished circulating plasma volume' is an unlikely explanation. Therefore, on the basis of the present observations, previously postulated causes of renal hypoperfusion in cirrhosis need revision.

The fundamental pathophysiological factor in functional renal failure in the late stage of cirrhosis (Kew, 1972) is renal vasoconstriction causing a decreased renal blood flow (Schroeder et al., 1967; Barnardo et al., 1970; Cohn et al., 1970;Epsteinetal., 1970). However, a diminished renal blood flow may be present even before there is clinical and laboratory evidence of impaired renal function (Kew et al., 1971). The pathogenesis of the renal hypoperfusion in cirrhosis has remained an enigma. Decreased renal blood flow has been demonstrated in patients with extrahepatic and presinusoidal portal hypertension and normal liver function (Kew et al., 1972). This, and several animal studies of experimental portal hypertension showing a decrease in the renal blood flow (Onnis et al., 1962; Onesti, 1971; Newsome et al., 1971; Kilcoyne et al., 1971, Gordon et al., 1973; Levy, 1974a, b), has drawnattention towards portal hypertension as a possible pathogenetic factor. The hypothesis is that either a direct portal-renal reflex, or splanchnic pooling, resulting in a decreased 'effective circulating plasma volume', may be the cause of the reduced renal blood flow. As the animal studies of experimental portal hypertension have all been in a non-steady state, and, as studies of the effect of portal decompression upon the renal circulation have not been performed in cirrhotic patients, the purpose of the present study

Received for publication 29 June 1976 was to evaluate the effect on renal haemodynamics in cirrhosis of normalising the portal pressure.

\section{Methods}

\section{PATIENTS}

Eleven patients with advanced cirrhosis of different aetiology and one patient with presinusoidal portal hypertension of unknown aetiology (possibly caused by arsenic medication) were examined immediately before and from two to seven months (mean 3.5 months) after a portal-systemic shunt was carried out. The interval allowed a new haemodynamic steady state to be established. Table 1 presents clinical and laboratory data on the patients, four females and eight males with a mean age of 50 years. The diagnoses were verified by liver biopsy. In all patients oesophageal varices were observed either by radiography or by oesophagoscopy, and the patients had experienced repeating bleeding episodes that were sufficiently severe to demand transfusions. Emergency operations were not performed. The patients were without clinical and electroencephalographic signs of hepatic encephalography at the time of investigation. Ascites and peripheral oedema were not present, but four patients received diuretics at the time of the first examination, and seven patients had been treated with diuretics at some time before the actual admission. Renal, cardiac, or hypertensive diseases were excluded by history, 
Table 1 Biochemical variables in 12 patients before and after portal-systemic shunt

\begin{tabular}{|c|c|c|c|c|c|c|c|c|c|}
\hline No. & Init. & Sex & $\begin{array}{l}\text { Age } \\
\text { (yr) }\end{array}$ & Diagnosis & $\begin{array}{l}\text { Serum bilirubin } \\
(<17 \mu \mathrm{mol} / 1)\end{array}$ & $\begin{array}{l}\text { Serum albumin } \\
(532-813 \mu \mathrm{mol} / \mathrm{l})\end{array}$ & $\begin{array}{l}\text { Prothrombin } \\
\text { time } \\
(85-115)\end{array}$ & $\begin{array}{l}\text { Galactose } \\
\text { elimination } \\
(1 \cdot 38-3 \cdot 50 \mathrm{mmol} / \\
\text { min })\end{array}$ & $\begin{array}{l}\text { Serum creatinine } \\
(<0.13 \mathrm{mmol} / \mathrm{l})\end{array}$ \\
\hline $\begin{array}{r}1 \\
2 \\
3 \\
4 \\
5 \\
6 \\
7 \\
8 \\
9 \\
10 \\
11 \\
12\end{array}$ & $\begin{array}{l}\text { EA } \\
\text { GB } \\
\text { KO } \\
\text { PL } \\
\text { LH } \\
\text { BS } \\
\text { SE } \\
\text { WH } \\
\text { KK } \\
\text { JP } \\
\text { KE } \\
\text { KH }\end{array}$ & $\begin{array}{l}\mathbf{F} \\
\mathbf{F} \\
\mathbf{F} \\
\mathbf{M} \\
\mathbf{F} \\
\mathbf{M} \\
\mathbf{M} \\
\mathbf{M} \\
\mathbf{M} \\
\mathbf{M} \\
\mathbf{M} \\
\mathbf{M}\end{array}$ & $\begin{array}{l}56 \\
59 \\
28 \\
57 \\
57 \\
37 \\
53 \\
51 \\
47 \\
72 \\
42 \\
43\end{array}$ & $\begin{array}{l}\text { AC } \\
\text { IC } \\
\text { PPH } \\
\text { AC } \\
\text { IC } \\
\text { AC } \\
\text { AC } \\
\text { AC } \\
\text { AC } \\
\text { IC } \\
\text { AC } \\
\text { AC }\end{array}$ & $\begin{array}{c}11-57 \\
55-59 \\
16-35 \\
10-10 \\
16-24 \\
43-22 \\
16-22 \\
31-19 \\
5-8 \\
14-71 \\
6-6 \\
14-20\end{array}$ & $\begin{array}{l}347-366 \\
420-307 \\
508-452 \\
473-446 \\
390-377 \\
462-446 \\
489-500 \\
418-445 \\
535-407 \\
530-393 \\
436-440 \\
463-421\end{array}$ & $\begin{array}{l}41-27 \\
60-39 \\
59-48 \\
68-71 \\
69-62 \\
83-78 \\
74-73 \\
57-65 \\
87-62 \\
72-50 \\
99-76 \\
65-57\end{array}$ & $\begin{array}{l}1 \cdot 48-- \\
1 \cdot 28-1 \cdot 03 \\
1 \cdot 68-1 \cdot 38 \\
3 \cdot 06-2 \cdot 54 \\
2 \cdot 11-1 \cdot 21 \\
1 \cdot 11-1 \cdot 16 \\
1 \cdot 12-1 \cdot 07 \\
1 \cdot 73-1 \cdot 74 \\
1 \cdot 75-1 \cdot 65 \\
1 \cdot 21-1 \cdot 03 \\
2 \cdot 32-2 \cdot 17 \\
1 \cdot 66-1 \cdot 49\end{array}$ & $\begin{array}{l}0.08-0.08 \\
0.08-0.06 \\
0.08-0.08 \\
0.08-0.07 \\
0.08-0.09 \\
0.12-0.09 \\
0.11-0.12 \\
0.09-0.08 \\
0.11-0.08 \\
0.10-0.08 \\
0.06-0.08 \\
0.09-0.08\end{array}$ \\
\hline Mean & & & 50 & & $\begin{array}{l}19-29 \\
P>0.05\end{array}$ & $\begin{array}{l}455-416 \\
P<0.05\end{array}$ & $\begin{array}{l}69-59 \\
P<0.01\end{array}$ & $\begin{array}{l}1.62-1.49 \\
P<0.02\end{array}$ & $\begin{array}{l}0.09-0.08 \\
P>0.05\end{array}$ \\
\hline
\end{tabular}

AC: alcoholic cirrhosis.

IC: idiopathic cirrhosis.

PPH: presinusoidal portal hypertension.

physical examination, and laboratory findings. At the time of the investigation the patients' hydration was normal and there were no abnormalities in their electrolyte status.

In two patients (cases 1 and 7) a splenorenal shunt, and in two patients (cases 2 and 11) a side-to-side portacaval shunt was performed. The rest of the patients had an end-to-side portacaval shunt. In two cases complications occurred. In case 7 a thrombosis of the shunt necessitated a splenorenal shunt nine days after the first operation. Normal function of this shunt was verified by coeliac angiography. Case 8 went into hepatic coma (grade II-III) after surgery. Later, sepsis with Escherichia coli occurred, during which the patient had gastrointestinal bleeding from a duodenal ulcer (seen at gastroscopy). The sepsis was treated with antibiotics, and the ulcer was treated conservatively. Both complications were eventually overcome.

A decrease in intrasplenic pressure or a radiographically open shunt was observed in all cases. However, in three cases a discrepancy between intrasplenic pressure decrease and radiographical findings was found. In case 4 a moderately increased intrasplenic pressure remained unchanged after surgery ( 23 to $22 \mathrm{~mm} \mathrm{Hg}$ ) despite a radiographically open shunt. In case 5 the intrasplenic pressure fell considerably, but not to normal levels ( 37 to $28 \mathrm{~mm} \mathrm{Hg}$ ). On the splenoportogram the shunt appeared open, but minimal oesophageal varices were still present. In case 11 the intrasplenic pressure fell to normal, but an open shunt was not visualised at the splenoportogram. None of the patients experienced bleeding from oesophageal varices during the interval between surgery and follow-up examination.

\section{TECHNIQUES}

The following programme was carried out before and after shunt operation. After fasting for eight hours the patients were premedicated with diazepam $10 \mathrm{mg}$. A right-sided cardiac catheterisation was performed, and subsequently the hepatic veins were catheterised in order to measure the wedged hepatic venous pressure. Then an Ödman catheter no. 6 was introduced percutaneously under local anaesthesia into a femoral artery, and under fluoroscopic control the tip was placed in the renal artery. Catheter position and possible reflux during injection was checked by injecting 5-10 ml 30\% Urografin. Renal blood flow was measured at least 10 minutes after Urografin was injected (Caldicott et al., 1970). The catheter was also used to sample arterial blood.

The cardiac output was measured according to the principle of Fick while the venous catheter was placed in the pulmonary artery.

Plasma and erythrocyte volume were measured with the Evans-Blue technique and 51-Cr tagged erythrocytes according to method A as recommended by the International Committee for Standardization in Hematology (1971). Hepatic function was estimated by measuring galactose elimination capacity (Tygstrup et al., 1963).

Renal blood flow (RBF) was measured by the ${ }^{133}$ Xenon washout method, as described by Ladefoged (1966). A bolus injection of 1.0-2.0 mCi ${ }^{133}$ Xenon (Amersham-XAS-11-P) dissolved in isotonic saline solution was injected into the renal artery, and the washout curve was followed with a scintillation detector placed below the kidney. The curve was registered through a Selektronik pulse-height analyser model 45-22 with an optimal 'window' for ${ }^{133}$ Xenon. Peak count was more than $3000 \mathrm{c} / \mathrm{s}$ and time constant $1 \mathrm{~s}$. Two washout curves were performed and the mean RBF was calculated from the average of the two initial slopes in a semi-logarithmic plot. The diffusion constant was corrected for 
haematocrit according to Ladefoged (1966).

Control values for RBF were obtained in eight patients, four males and four females, aged 23 to 44 years, who were undergoing cardiac catheterisation for suspected but unconfirmed cardiac disease.

In all patients a splenoportogram with measurement of the intrasplenic pressure was performed. Unless otherwise stated, statistical analysis of observations before and after shunt operation was done by the method of paired comparison.

\section{Results}

As shown in the Figure the RBF was significantly lower in the patients with portal hypertension than in the controls, $1.95 \mathrm{ml} / \mathrm{g} / \mathrm{min}$ and $3.50 \mathrm{ml} / \mathrm{g} / \mathrm{min}$, respectively $(\mathrm{P}<0.01)$, (rank sum test, Mann-Whitney),

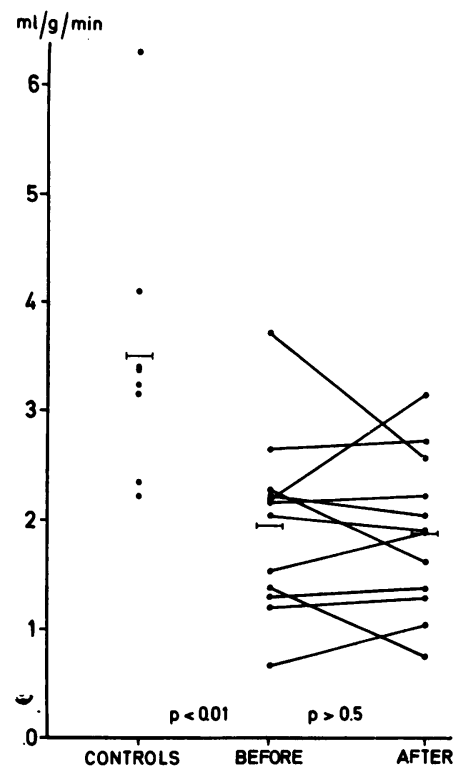

Figure Mean renal blood flow in eight controls and in 12 patients with portal hypertension before and after decompression operation. even when the age difference between the two groups was considered (Hollenberg et al., 1974). After the portal-systemic shunt operation the mean RBF was $1.88 \mathrm{ml} / \mathrm{g} / \mathrm{min}$ and thus unchanged. The haemodynamic consequences of portal-systemic shunting were independent of shunt type or the aetiology of portal hypertension.

Some of the haemodynamic variables found before and after portal-systemic shunt are shown in Table 2. The plasma volume and, to a lesser extent, the blood volume were significantly greater before the shunt than could be predicted from age, sex, and weight (Documenta Geigy, Scientific Tables, 1968), mean $3.931\left(59.5 \mathrm{ml} / \mathrm{kg}\right.$ or $\left.2.191 / \mathrm{m}^{2}\right)$, predicted $2.851(\mathrm{P}<0.01)$ and $5.681(84.5 \mathrm{ml} / \mathrm{kg}$ or $\left.3.17 \mathrm{l} / \mathrm{m}^{2}\right)$ predicted $4.841(\mathrm{P}<0.05)$. No significant change was seen after shunt operation ( $P>0.05)$.

The cardiac output increased significantly from a mean value $5.681 / \mathrm{min}\left(3.09 \mathrm{l} / \mathrm{min} / \mathrm{m}^{2}\right)$ to 6.76 $1 / \mathrm{min}\left(3.801 / \mathrm{min} / \mathrm{m}^{2}\right)(\mathrm{P}<0.01)$. This was exclusively due to an increase in the stroke volume from 72 to $92 \mathrm{ml}$.

The mean arterial blood pressure decreased from 87 to $82 \mathrm{~mm} \mathrm{Hg}(\mathrm{P}>0.05)$, while the total peripheral resistance fell significantly from a mean of 1390 to 1048 dyne s cm ${ }^{-5}(P<0.05)$.

After shunt surgery the intrasplenic pressure fell significantly from an average of 29 to $18 \mathrm{~mm} \mathrm{Hg}$ $(\mathbf{P}<0.001)$. The wedged hepatic venous pressure in the patients with cirrhosis receiving an end-to-side portacaval shunt is shown in Table 2 . The pressure fell from 24.3 to $20.5 \mathrm{~mm} \mathrm{Hg}(\mathrm{P}<0.001)$. In the two patients receiving a side-to-side shunt the wedged hepatic venous pressure fell from 23 to 14 and 22 to $14 \mathrm{~mm} \mathrm{Hg}$ respectively; thus in both cases this pressure became practically normal. Pressure in one patient with a splenorenal shunt fell from 27 to $21 \mathrm{~mm} \mathrm{Hg}$.

As can be seen in Table 1 the shunting caused a minor but significant decrease in liver function as evaluated by galactose elimination capacity $(P<0.02)$, while all patients had normal renal function before and after shunt as judged by the serum creatinine level.

Table 2 Effect of portal-systemic shunt on haemodynamic parameters

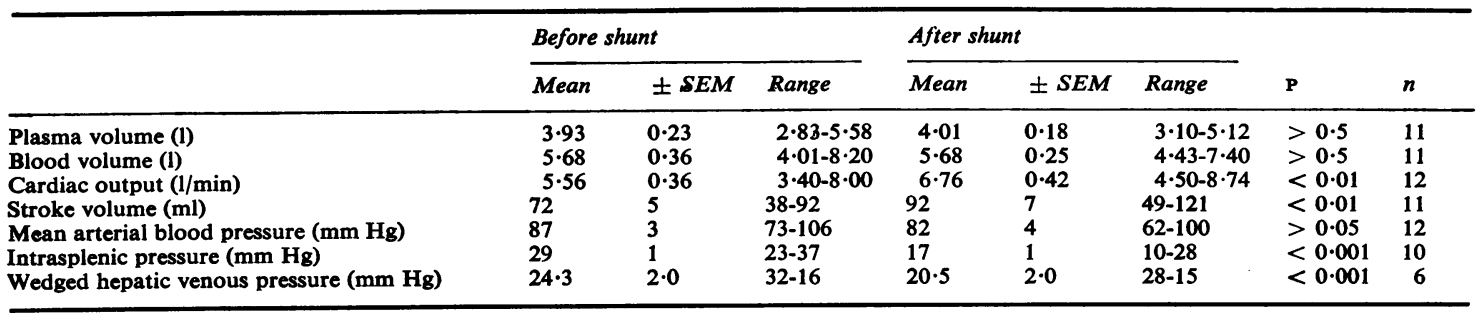




\section{Discussion}

A decrease in portal pressure and a more hyperkinetic systemic circulation was the result of shunt operation, while changes in an initially low renal perfusion failed to appear.

The high plasma and blood volume and larger than normal cardiac output before shunt surgery are in agreement with previous studies (Murray et al., 1958; Mashford et al., 1962; Lieberman et al., 1967).

Earlier studies have disclosed an increase in cardiac output and an unchanged or slightly decreased plasma volume after shunt operation. Foda et al. (1974) observed an increase in cardiac index from 4.78 to $6.761 / \mathrm{min} / \mathrm{m}^{2}$, and Gordon and Del Guercio (1972) found a parallel increase in cardiac index after shunt surgery in an initially normodynamic group of patients, while in an initially hyperdynamic group such an increase was absent. This difference does not appear in our material. Like us, Foda et al. (1964) found no changes in plasma volume or blood volume, whereas Lieberman and Reynolds (1967) found a decrease in these variables after shunting.

The mean RBF in the eight controls of this study corresponds well with the findings of Hollenberg et al. (1974) for this age group. The subnormal mean RBF in cirrhotic patients without signs of renal failure is similar to the results found by Kew and coworkers (1971) using the same method for determining the RBF in a comparable sample of patients.

$A$ decreased RBF in cirrhosis as observed in the present material is caused by renal vasoconstriction (Barnardo et al., 1970), but how this is initiated has not yet been established. The hypothesis that a raised portal pressure per se causes renal hypoperfusion is supported from animal studies (Onnis et al., 1962; Hanback, 1970; Onesti 1971; Newsome et al., 1971; Kilcoyne et al., 1971; Gordon et al., 1973, Levy, 1974a). However, in these studies the experimental increase in portal pressure has been acute, so that it was not possible for a new circulatory steady state to take place. On the other hand, the observation that RBF is reduced in patients with extrahepatic portal hypertension in a steady state situation supported this view (Kew et al., 1972). However, the present study does not establish a relationship between portal decompression and recovery of renal perfusion. In studying the effect of blocking hepatic venous outflow by histamine injection, Levy found a decrease of renal blood flow which could not be produced by a similar rise of portal venous pressure by portal vein constriction. He therefore postulated that the rise not in portal but in intrahepatic pressure caused renal hypo- perfusion (Levy, 1974b). That a reduction in RBF in cirrhotic patients is caused by an increase in intrahepatic rather than in portal pressure is a possibility. However, unchanged low RBF in two patients receiving side-to-side portacaval shunt, which also normalised the raised sinusoidal pressure does not support this view.

It has been suggested that a low 'effective circulating plasma volume' caused by splanchnic pooling is responsible for the low RBF seen in cirrhosis (Vlahcevic et al., 1965; Tristani and Cohn, 1967). The reports on the effect of portacaval anastomosis on functional renal failure in cirrhosis are conflicting, ranging from claims of direct improvement (Schroeder et al., 1970) to claims of initiation of the syndrome (Cohn, 1964; Garrett et al., 1970), although RBF has not been measured in these cases. Schroeder suggested, after observing improvement in diuresis and creatinine clearance in one patient with functional renal failure who received a shunt, that this caused a redistribution in plasma volume, so normalising the 'effective circulating plasma volume' and leading to improved renal function. The portalsystemic shunt should be capable of relieving the splanchnic pooling. If a decreased 'effective circulating plasma volume' is a reality, it may be speculated that a fraction of the plasma volume before shunt surgery is trapped in the splanchnic area, and after surgery runs idle in the shunt and in this way is equally ineffective in the circulation.

Vasoactive amines have been incriminated in the pathogenesis of the reduction of RBF in cirrhosis (Epstein et al., 1970; Fischer and Baldessarini, 1971). Speculation has been concentrated on the idea that such vasoactive agents are produced in, or not activated in, the sick liver. Correlation between endotoxins and renal impairment in acute liver failure has been recorded (Wilkinson et al., 1974). Epstein and his colleagues found irregularities in their ${ }^{133}$ Xenon washout curves which they attributed to circulating vasoactive amines. Since these vasoactive agents are thought to originate from the gut, a direct bypass via a portal-systemic shunt probably makes them more accessible to the target organ(s). The fact that, in the present study, the reduction in RBF did not decrease further in spite of unimproved or decreased liver function from first to second examination, seems to contradict this hypothesis, unless maximal detoxification is already taking place in the liver. However, vasoactive agents originating elsewhere-for example, via incomplete hormone catabolism, perhaps in the liver itself-are more likely to be responsible for the renal hypoperfusion.

On the basis of the present results, previously suggested causes for renal hypoperfusion in cirrhosis must be revised. A direct portal-renal, neural or 
humoral, reflex mechanism seems absent. A diminished 'effective circulating plasma volume' is unlikely. Vasoactive amines or endotoxins from the intestines seem less probable, while agents with similar effect originated elsewhere, perhaps in the poorly functioning liver itself, are a possibility.

This study has been supported by a grant from the Danish Research Council.

\section{References}

Barnardo, D. E., Baldus, W. P., and Maher, F. T. (1970). Effects of dopamine on renal function in patients with cirrhosis. Gastroenterology, 58, 524-531.

Caldicott, W. J. H., Hollenberg, N. K., and Abrams, H. L. (1970). Characteristics of respons of renal vascular bed to contrast media. Evidence for vasoconstruction induced by renin-angiotensin system. Investigative Radiology, 5, 539-547.

Cohn, J. N. (1964). Hepatorenal failure following portacaval shunt: hemodynamic considerations and the application of ascitic fluid infusion. Medical Annals of the District of Columbia, 33, 567-569, 594.

Cohn, J. N., Tristani, F. E., and Khatri, I. M. (1970). Renal vasodilator therapy in the hepatorenal syndrome. Medical Annals of the District of Columbia, 39, 1-7.

Epstein, M., Berk, D. P., Hollenberg, N. K., Adams, D. F. Chalmers, T. C., Abrams, H. L., and Merrill, J. P. (1970). Renal failure in the patient with cirrhosis. American Journal of Medicine, 49, 175-185.

Fischer, J. E., and Baldessarini, R. J. (1971). False neurotransmitters and hepatic failure. Lancet, $2,75$.

Foda, H., Badawi, H., and Salah, M. (1964). Circulatory hemodynamics before and after portocaval shunt operation in bilharzial hepatic fibrosis. American Heart Journal, 67, 295-303.

Garrett, J. C., Voorhees, A. B. Jr, and Sommers, S. C. (1970). Renal failure following portasystemic shunt in patients with cirrhosis of the liver. Annals of Surgery, 172, 218-225.

Gordon, S. J., Bazzato, G., Onesti, G., and Kowlessar, O. D. (1973). Experimental portal hypertension: Effect on renal function (Abstract). Gastroenterology, 64, 735.

Gordon, M. J., and Del Guercio, L. R. M. (1972). Late effects of portal systemic shunting procedures on cardiorespiratory dynamics in man. Annals of Surgery, 176, 672-679.

Hanback, L. D. Jr (1970). Renal hemodynamics following minimal hepatic outflow block. Surgical Forum, 21, 364-366.

Hollenberg, N. K., Adams, D. F., Solomon, H. S., Rashid, A., Abrams, H. L., and Merrill, J. P. (1974). Senescence and the renal vasculature in normal man. Circulation Research, 34, 309-316

International Committee for Standardization in Hematology. (1971). Recommended methods for radioisotope red cell survival studies. Blood, 38, 378.,

Kew, M. (1972). Renal changes in cirrhosis. Gut, 13, 748-753.

Kew, M. C., Limbrick, C., Varma, R. R., and Sherlock, S. (1972). Renal and intrarenal blood flow in non-cirrhotic portal hypertension. Gut, 13, 763-767.

Kew, M. C., Varma, R. R., Williams, H. S., Brunt, P. W., Hourigan, K. J., and Sherlock, S. (1971). Renal and intrarenal bloodflow in cirrhosis of the liver. Lancet, 2, 504-509.

Kilcoyne, M. M., and Cannon, P. J. (1971). Influence of thoracic caval occlusion on intrarenal blood flow distribution and sodium excretion. American Journal of Physiology, $220,1220-1230$.

Ladefoged, J. (1966). Measurements of the renal blood flow in man with the ${ }^{133}$ Xenon wash-out technique. Scandinavian Journal of Clinical and Laboratory Investigation, 18, 299-315.

Levy, M. (1974a). Renal function during acute graded elevation of portal venous pressure. American Journal of Physiology, 227, 1084-1087.

Levy, M. (1974b). Renal function in dogs with acute selective hepatic venous outflow block. American Journal of Physiology, 227, 1074-1083.

Lieberman, F. L., and Reynolds, T. B. (1967). Plasma volume in cirrhosis of the liver: its relation to portal hypertension, ascites, and renal failure. Journal of Clinical Investigation, 46, 1297-1308.

Mashford, M. L., Mahon, W. A., and Chalmers, T. C. (1962). Studies of the cardiovascular system in the hypotension of liver failure. New England Journal of Medicine, 267, 1071-1074.

Murray, J. F., Dawson, A. M., and Sherlock, S. (1958). Circulatory changes in chronic liver disease. American Journal of Medicine, 24, 358-367.

Newsome, H. H. Jr, Kafka, M. S., and Bartter, F. C. (1971). Intrarenal blood flow in dogs with constriction of the inferior thoracic vena cava. American Journal of Physiology, 221, 48-52.

Onesti, G. (1971). Renal effects of acute portal vein constriction. American Society for Nephrology, p. 59.

Onnis, M., Shumacker, H. B. Jr, and Bounous, G. (1962). Response to occlusion of the portal vein. Archives of Surgery, 85, 897-900.

Schroeder, E. T., Numann, P. J., and Chamberlain, B. E. (1970). Functional renal failure in cirrhosis. Annals of Internal Medicine, 72, 923-928.

Schroeder, E. T., Shear, L., Sancetta, S. M., and Gabuzda, G. J. (1967). Renal failure in patients with cirrhosis of the liver. III. Evaluation of intrarenal blood flow by paraaminohippurate extraction and response to angiotensin. American Journal of Medicine, 43, 887-896.

Tristani, F. E., and Cohn, J. N. (1967). Systemic and renal hemodynamics in oliguric hepatic failure: effect of volume expansion. Journal of Clinical Investigation, 46, 1894-1906.

Tygstrup, N. (1966). Determination of the hepatic galactose elimination capacity after a single intravenous injection in man. Acta Physiologica Scandinavica, 58, 162172.

Vlahcevic, Z. R., Adham, N. F., Jick, H., Moore, E. W., and Chalmers, T. C. (1965). Renal effects of acute expansion of plasma volume in cirrhosis. New England Journal of Medicine, 272, 387-391.

Wilkinson, S. P., Gazzard, B. G., Arroyo, V., Moodie, H.. and Williams, R. (1974). Relation of renal impairment and haemorrhagic diathesis to endotoxaemia in fulminant hepatic failure. Lancet, 1, 521-524. 